\title{
Erratum to: Recycled Aggregate Concrete Structures
}

\section{Erratum to: \\ Recycled Aggregate Concrete Structures, Springer Tracts in Civil Engineering, https://doi.org/10.1007/978-3-662-53987-3}

In the original version of the book, the belated corrections from author for all the chapters have been incorporated.

Figures and Equations are updated in book.

The updated online versions of these chapters can be found at https://doi.org/10.1007/978-3-662-53987-3 